\title{
Sensitivity Analysis of AODV Protocol Regarding Forwarding Probability
}

\author{
V. Kanakaris ${ }^{1}$, D. $\mathrm{Ndzi}^{1}$ and G.A. Papakostas ${ }^{2}$ \\ ${ }^{1}$ School of Engineering, University of Portsmouth, United Kingdom \\ ${ }^{2}$ Dept. of Computer and Informatics Engineering, Eastern Macedonia and Thrace Institute of \\ Technology, Kavala, Greece \\ Email: \{venetis.kanakaris, david.ndzi,\}@ port.ac.uk, gpapak@teikav.edu.gr
}

\begin{abstract}
This paper focuses on how the probability can affect on the Ad Hoc routing protocols and especially on AODV regarding energy consumption. The evaluation of the performance of AODV with different Message Forwarding Probability based on the mechanism that AODV protocol uses in order to forward the message in all nodes of the network. The evaluation of AODV protocol was carried out using Network Simulator-2 (NS-2), on ten scales of probability. The outcome of this evaluation showed that using smaller probability than that of the original AODV protocol has, we can have better results on power consumption.
\end{abstract}

Keywords - Ad-hoc networks, AODV, power consumption, forwarding probability, routing protocols

\section{Introduction}

Mobile Ad Hoc networks (MANETs) are rapidly evolving as an important research area of wireless mobility. MANET is a dynamically self-organizing network without supported infrastructure or central administrator. The main characteristic of MANET is that the network topology changes very fast and unpredictably in which mobile nodes moves to and from other wireless networks without any fixed access point where routers and hosts move constantly, so the topology dynamically changed [1]. Therefore, frequent change of the network topology is rough challenge for many important issues, such as increasing overhead, limited bandwidth and energy loss. In order to reduce the power consumption on the AODV protocol we suggest a heuristic method that reduces the routing messages and therefore we get low routing load and low energy consumption. By this way we propose to change dynamically the probability of route message forwarding and therefore to reduce the average consumed energy of all nodes, the number of dropped packets of all the nodes, the total amount of data received/transmitted from the nodes over time [1][4]. 
There are many protocols [5] that have been developed in the last years for non-centralised networks, e.g. Temporally Order Routing protocol (TORA) [5], Dynamic Source Routing protocol (DSR) [6], On-Demand Distance Vector protocol (AODV) [7], Destination-Sequenced Distance-Vector Routing protocol (DSDV) [8], Optimized Link State Routing protocol (OLSR) [9]. In all the above routing protocols some need to keep the routing information from every node to any other node in the network, while some other try to create routes on an on-demand situations. By keeping information regarding the route needs an enormous portion of network capacity, although most of this information is used very rare. On the other hand, the on-demand routing protocols there is a lag on creating the route and while trying to create the route an overhead caused and therefore energy depletion.

This research focuses on AODV routing protocol and specifically aimed at limiting the routing message overhead and hence power consumption and prolonging battery life. This will be accomplished by decreasing any redundant broadcasting from the nodes with predefined probability.

\section{AODV Protocol - A Brief Description}

AODV Error! Reference source not found. is a reactive routing protocol that establishes a route to a destination on-demand. That is, a route is established only when it is required by a source node for transmitting data packets. This is beneficial in mobile ad-hoc networks since up-to-date knowledge of all routes from every node implies large communication overhead. The routing mechanism of AODV consists of two processes:

- Route discovery

- Route maintenance.

When the source node wants to send information to a node and there in no valid route, it start the process to discover the route by sending the route request (RREQ) packet to all nodes using a simple flooding technique [10] (Fig. 1).

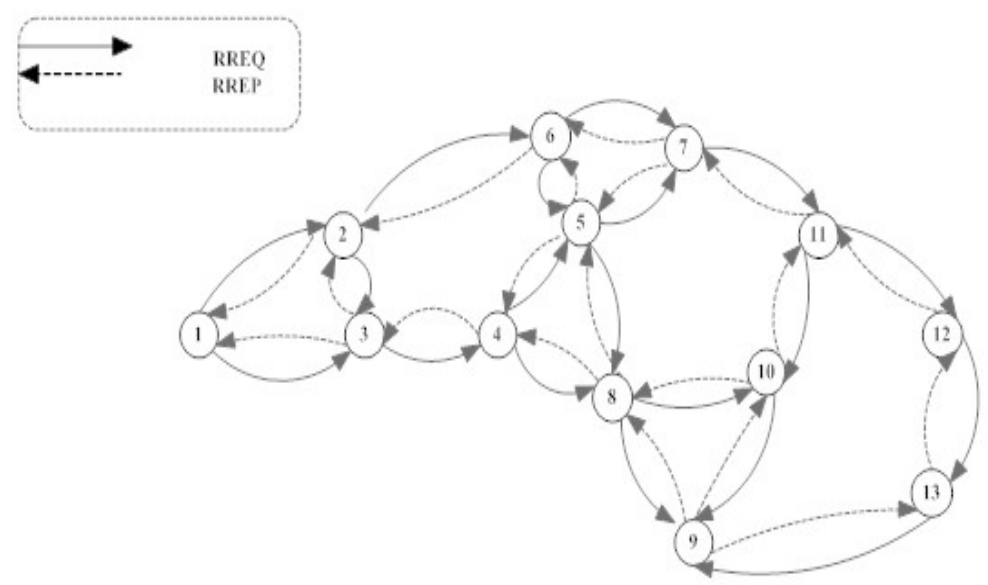

Figure 1: A typical AODV network of nodes. 
The RREQ packet has the following main parts:

1. Source identifier,

2. Destination identifier,

3. Source sequence number,

4. Destination sequence number (ensure that routes are loop-free and contain the most recent route information)

5. Broadcast identifier (Request ID)

6. Time-to-live

\subsection{Route Request Mechanism (RREQ)}

In AODV the messages that are broadcasted include Route Request (RREQ), Route Reply (RREP), and Route Error (RERR). These are saved in routing table entries in the form of concrete status information for destinations of interest. Each entry in the routing table has the latest information such as sequential serial number for the destination. The specific number is called destination sequence number which is updated when a node receives new information from RREQ, RREP, or RERR messages about the destination. If each node in the network is denoted by $\mathrm{N}$ and there are $\mathrm{m}$ nodes in the network then $\operatorname{sq}\left(\mathrm{N}_{\mathrm{i}}\right)$ is the sequential number of node $\mathrm{i}$.

A destination node increases the sequence number in the following cases:

- Before it starts the search process for new route, it increases its sequence number. In this way, it protects against possible conflicts with previously defined routes to the source. $s q\left(N_{i}\right)=s q\left(N_{i}\right)+1$

- When it intends to start sending a RREP, it has to renew its own sequence number to the maximum of its current sequence number $s q\left(N_{i}\right)$ and the destination sequence number $s q\left(N_{j}\right)$ in the RREQ packet.

$$
\begin{aligned}
& \text { if } \mathrm{sq}\left(\mathrm{N}_{\mathrm{i}}\right) \leq \mathrm{sq}\left(\mathrm{N}_{\mathrm{j}}\right) \text { then } \mathrm{sq}\left(\mathrm{N}_{\mathrm{i}}\right)=\mathrm{sq}\left(\mathrm{N}_{\mathrm{j}}\right) \mathrm{i}, \mathrm{j}=1 \ldots . \mathrm{m} \\
& \text { if } \mathrm{sq}\left(\mathrm{N}_{\mathrm{j}}\right) \leq \mathrm{sq}\left(\mathrm{N}_{\mathrm{i}}\right) \text { then } \mathrm{sq}\left(\mathrm{N}_{j}\right)=\mathrm{sq}\left(\mathrm{N}_{\mathrm{i}}\right) \mathrm{i}, \mathrm{j}=1 \ldots . \mathrm{m}
\end{aligned}
$$

- A destination node can increment the sequence number $\mathrm{sq}\left(\mathrm{N}_{\mathrm{j}}\right)$ by one if one of the three following statements is true:

$$
s q\left(N_{j}\right)=s q\left(N_{j}\right)+1\left\{\begin{array}{c}
N_{i} \neq N_{j} \\
N e w s q\left(N_{j}\right) \\
\text { No valid path }
\end{array}\right.
$$




\subsection{The routing table entries and lists of precursor}

When a node receives a control packet (routing packet) from its neighbour, or creates, or renews a path to a particular destination or subnet, it searches in its routing table for a valid entry for this destination. If there is no record in the table it creates a new one. The sequence number is defined by the information contained in the control packet.

The sequential number is renewed at the following cases:

- When the value is higher than that in the recorded routing table, that is:

if $s q\left(N_{i}\right)$ is sequence number of node $\mathrm{i}$ in the routing table, if it receives a new number $s q\left(N_{i}\right)^{\prime}>s q\left(N_{i}\right)$ then $s q\left(N_{i}\right)^{\prime}=s q\left(N_{i}\right)$

- When the value is the same as in the record, but the sum of the required steps (number of hops) is smaller than that in the table, that is:

$\sum_{j=i+1}^{k} \operatorname{hop} C\left(N_{i,} N_{j}\right)<\sum_{j=i+1}^{l} \operatorname{hop} C\left(N_{i,} N_{j}\right), k \leq l \leq m$

where $\sum_{j=i+1}^{l} h o p\left(N_{i}, N_{j}\right)=n+1$ is the number of hopes from node $N_{i}$ to node $N_{j}$ that is currently stored in the table and $\sum_{j=i+1}^{k} \operatorname{hop} C\left(N_{i}, N_{j}\right)=n$ is the updated number of hops from node $N_{i}$ to node $N_{j}$

- When the sequential number is unknown $s q\left(N_{i}\right)=s q\left(N_{i}\right)+1$

For each valid route maintained in a routing table, a list of precursors is maintained, i.e. intermediate nodes that can forward packets along the route. The precursors are notified by the node if it loses a link route.

\subsection{Route Request Query}

A node distributes a RREQ packet if there is a route request to a destination. This can happen if the destination is not known in advance or if a path was invalid or becomes inactive. The value of the destination sequential number that is copied to a routing table, is contained in the packet and is associated with the last number received. The exact number is increased when a priority RREQs messages is sent.

If $s q\left(N_{j}\right)$, is the sequence number to the destination node $j$, then: $s q\left(N_{j}\right)=s q\left(N_{j}\right)+n_{\text {priority }}$ where $\mathrm{n}_{\text {priority }}$ is the priority number of received RREQs message. 
The $R R E Q_{I D}$ field is increased from a value of 1 except the last $R R E Q_{I D}$ that is used by the current node. Each node maintains only one $R R E Q_{I D}$, the source node enters the $R R E Q_{I D}$ and the IP address of the source of RREQ for PATH_DISCOVERY_TIME. PATH_DISCOVERY_TIME procedure of the RREQ mechanism, that is used to prevent nodes from responding to the same RREQ message it has already processed.

If $\left(R R E Q_{I D}\right)_{i}=1$ then $\left(R R E Q_{I D}\right)_{n+1}=\left(R R E Q_{I D}\right)_{n}+1, \mathrm{n}=\mathrm{i}, . .1,1 \leq n$ where $\mathrm{i}$ is the number of current node and RREQ ${ }_{I D}$ field is to be used by the current node.

The communication between a source and a destination is duplex. That means that the destination has to have a route to the source. To achieve this efficiently, the creation of a RREP by an intermediate node is accompanied about the route to the source.

The RREP packet has the following main parts:

1. Source identifier

2. Destination identifier

3. Destination sequence number

4. Hop Count

5. Lifetime

Every intermediate node that forwards a RREQ packet creates a reverse route back to the source node by imprinting the next hop information in its routing table. When the RREQ packet finds the destination node or an intermediate node with a valid route, the node responds by sending a route reply (RREP) packet to the source using the reverse route. The validity of a route at the intermediate nodes is determined by comparing its sequence number with the destination sequence number. Each node that takes part in forwarding RREP packets back to the source creates a forward route to the destination by saving the next hop information in the routing table. Nodes along the path from source to destination are not required to have knowledge of all nodes other than the next hop node to the source and destination.

In addition AODV also has a route maintenance process. After the route discovery process and the discovered route is in use, the intermediate nodes together with the active route maintain an up-to-date list of their one-hop neighbours using a periodic exchange of "hello" packets. If the route becomes inactive, e.g. no battery life, a timer is activated to inform the network about the expiration of this route. When the routing agent of a node becomes aware of a link breakage for an active route, a Route Error (RERR) packet is generated and disseminated to the appropriate nodes participating in the route's formation. When the RERR packet reaches the source node a new route discovery is initiated. 
AODV uses a simple flooding method for route discovery where a source node transmits to all nodes in the vicinity. Each node checks whether it has received this message before. If it had, then the message will be dropped, if not then the message is re-transmitted to all neighbouring nodes. This process continues until all nodes get the message. However, this method increases the network traffic and depletes battery power.

A probabilistic message forwarding scheme (a forwarding scheme that uses a probability to choose the number of nodes to forward the messages) could be used that will reduce the routing message overhead and hence power consumption of AODV. This can be achieved by reducing any redundant broadcasting from the nodes with a predetermined probability. The most important factor in this scheme is the forwarding probability.

The NS-2 simulation software package that will be used for this research follows a specific pattern to calculate the power consumption. The Energy Model [11] starts with an initial energy, $P_{i}$, for each node which decreases with every packet that is transmitted. Let the reduction in power when transmitting and receiving be denoted by DTxE and DRxE, respectively. These values depend on the amount of power spent per unit time when transmitting, $P_{t x}$, and receiving, $P_{r x}$, and the time duration in each of these modes, $t_{t x}$ and $t_{r x}$. When the energy level becomes zero, then no more packets can be transmitted or received by the node.

\section{Proposed AODV Modification}

This paper proposes the following modifications to AODV protocol:

Assume that there are $N$ nodes in the network and $n$ is the number of nodes in the neighbourhood of a transmitting node.

In normal AODV route message forwarding, if a node is forwarding a packet then all the n neighbours will try to forward the message again. If $\mathrm{P}_{\mathrm{rx}}$ is the power consumed when receiving a route control message and $\mathrm{P}_{\mathrm{tx}}$ is the power consumed when forwarding a route control message, then all $n$ nodes will spend $\mathrm{P}_{\mathrm{s}}$ power equal to $\mathrm{P}_{\mathrm{s}}=\left(\mathrm{P}_{\mathrm{tx}}+\mathrm{P}_{\mathrm{rx}}\right) * n$.

If $\mathrm{P}_{i}$ is the sum of total initial power of the $\mathrm{n}$ neighbouring nodes, then the total remaining power of the neighbouring node remaining is

$$
\mathrm{P}_{\text {total }}=\left(\mathrm{P}_{i}-\mathrm{P}_{\mathrm{s}}\right)
$$


If probabilistic route message forwarding scheme is used, for example, assume that only $50 \%$ of the nodes are allowed to forward the message at any instance based on probability 0.5 , this means that only $\frac{n}{2}$ nodes will receive and forward the message. This means that the network residual power will be

$$
\mathrm{P}_{\text {total }}=\left(\mathrm{P}_{i}-\frac{P_{s}}{2}\right)
$$

If we compare Eq.(1) and Eq.(2), the latter will preserve the battery power and double the lifetime of the network. For the development and application of the probabilistic approach, the author will investigate the application of Bayesian principles in formulating the routing probability [12] .

Studies will also be carried out to modify AODV to use density based probabilistic route message forwarding with dynamic probability so that only a certain percentage of the n neighbours will forward the message based on the density of its neighbours. And if the density is high then $\mathrm{P}_{\text {total }}$ is expected to be much higher than that of Eq.(2). The following Table 1, illustrates the nomenclature used in the analysis.

Table 1 - Nomenclature.

\begin{tabular}{|l|l|}
\hline Field & Description \\
\hline Rtx & Power consumed during forwarding a route control message \\
\hline Rrx & Power consumed during receiving a route control message \\
\hline $\mathrm{P}_{\text {total }}$ & $\begin{array}{l}\text { The sum of total remaining power of the neighbouring nodes } \\
\text { node }\end{array}$ \\
\hline $\mathrm{P}_{\mathrm{i}}$ & The sum of total initial powers of the n neighbours \\
\hline $\mathrm{P}_{\mathrm{s}}$ & The total power of all nodes \\
\hline $\mathrm{n}$ & Number of nodes in the neighbourhood \\
\hline
\end{tabular}

\section{Simulations Configuration}

The aim of these simulations is to analyze the AODV protocol which has originally 1.0 probability by comparing it in different message forwarding probability (0.1 to 1.0$)$ for its efficiency in terms of power as well as throughput. This has been made by measuring the energy with respect to different network size and taking into consideration the remaining battery power. The simulation tool that has been used in this study is ns2 [11]. 


\subsection{Parameters of the Simulation}

The following Table 2 summarizes the settings of the parameters applied in all the conducted simulations.

Table 2 - Parameters settings.

\begin{tabular}{|l|l|}
\hline The Protocol & $\begin{array}{l}\text { Probabilistic RREQ Message } \\
\text { Forwarding Protocol }\end{array}$ \\
\hline Topographical Area & $600 \mathrm{~m}$ x $600 \mathrm{~m}$ \\
\hline Number of Nodes & $10,20,30,40,50,60,70,80,90,100$ \\
\hline Mobility & $1 \mathrm{~m} / \mathrm{s}$ \\
\hline Probability & $\begin{array}{l}0.10,0.20,0.30,0.40,0.50,0.60, \\
0.70,0.80,0.90,1.0\end{array}$ \\
\hline $\begin{array}{l}\text { Radio-propagation } \\
\text { model }\end{array}$ & TwoRayGround \\
\hline Network interface type & WirelessPhy \\
\hline MAC type & $802 \_11$ \\
\hline Interface queue type & DropTail/PriQueue \\
\hline Antenna model & OmniAntenna \\
\hline Total Simulation Time & 100 sec \\
\hline $\begin{array}{l}\text { Transport /Traffic } \\
\text { Type }\end{array}$ & CBR over UDP \\
\hline TxPower of the nodes & 0.1819 watts \\
\hline RxPower of the nodes & 0.0501 watts \\
\hline IdlePower of the nodes & 0.0350 watts \\
\hline Initial energy of the & 1000.0 Joules \\
\hline nodes & 10 \\
\hline Reenario Simulation & \\
\hline
\end{tabular}


The performance of the AODV routing protocol with network size varying from 10 to 100 nodes with probability ( 0.10 to 1.0$)$ have been assessed. In all the cases, only two senders Constant Bit-rate (CBR) over User Datagram Protocol (UDP) and two receivers (null sink) have been used.

\subsection{Indices considered for Evaluation [13]}

The following performance indices are used in the evaluations that follows:

\section{Number of Packets dropped:}

This is the number of data packets that are not successfully sent to the destination during the transmission. In this study the time versus number of packets dropped have been calculated.

\section{Consumed Power:}

The number of nodes in the network versus average consumed battery power is considered as a metric.

\section{Throughput:}

The throughput metric measures how well the network can constantly provide data to the sink. Throughput is the number of packet arriving at the sink per ms.

\section{MAC Load:}

This is the ratio of the number of MAC layer messages propagated by every node in the network to the number of data packets successfully delivered to all destination nodes. In other words, the MAC load is the average number of MAC messages generated to each data packet successfully delivered to the destination.

\section{Dropped Packets:}

This is the number of nodes in the network versus agent level total dropped packet.

\section{Network Load:}

Provides information regarding the availability and the reliability of the network.

\section{Results and Analysis}

Figure 2 shows that in small and medium size networks the consumed power, using 0.1 and 0.5 RREQ forwarding probability, is less than using 1.0 RREQ forwarding probability, which uses the original AODV protocol. For large networks, RREQ forwarding probability of 0.6 and 0.8 provides very good results as it consumes less energy than the other RREQ forwarding probabilities.

Figure 3 shows that for large networks and all network sizes, RREQ forwarding probability of 0.8 provides, on average, the best performance, with a battery power consumption of $2.845 \times 10^{3}$ Joules. . 
This is followed by a network with a RREQ forwarding probability of 0.6 which achieves an average battery power consumption of $2.853 \times 10^{3}$ Joules.

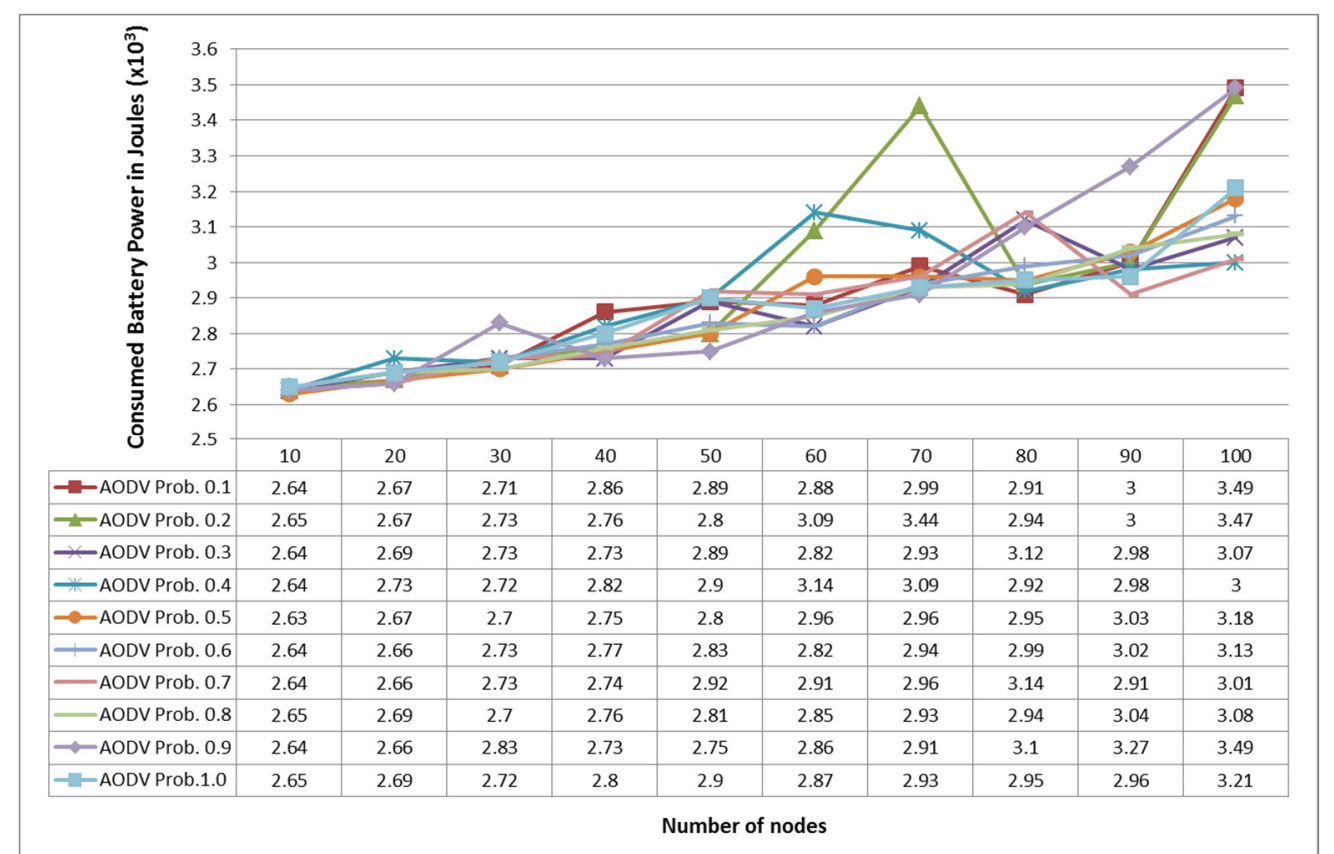

Figure 2: Average consumed power against the number of nodes for different RREQ Forwarding Probability.

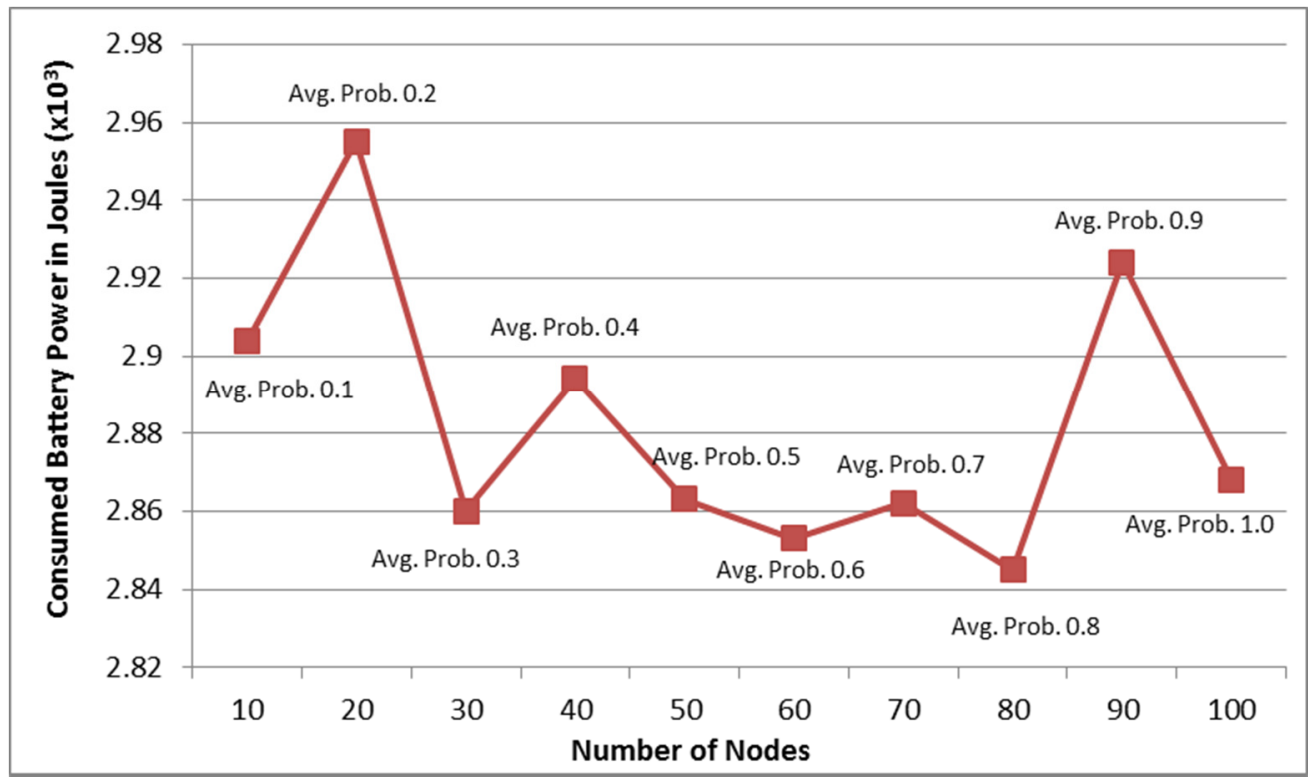

Figure 3: The average consumed power against the number of nodes for average RREQ Forwarding Probability.

Figure 4 shows that in small and medium size networks, RREQ forwarding probability of 0.1 performs even better than networks in which all nodes broadcast the RREQ (probability of 1.0). For large networks and all network sizes, RREQ forwarding probability of 0.8 provides, on average, the best 
performance with a throughput of $5.405 \mathrm{kbps}$. This is followed by a network with a RREQ forwarding probability of 0.4 which achieves an average throughput of $5.248 \mathrm{kbps}$. This shows that network performance is not adversely affected by reducing the number of nodes that rebroadcast messages.

Figure 5 shows that for large networks, RREQ forwarding probability of 0.8 provides, on average, the best performance, with a throughput $5.405 \mathrm{kbps}$. This is followed by a network with a RREQ forwarding probability of 0.4 which achieves an average throughput $5.276 \mathrm{kbps}$.

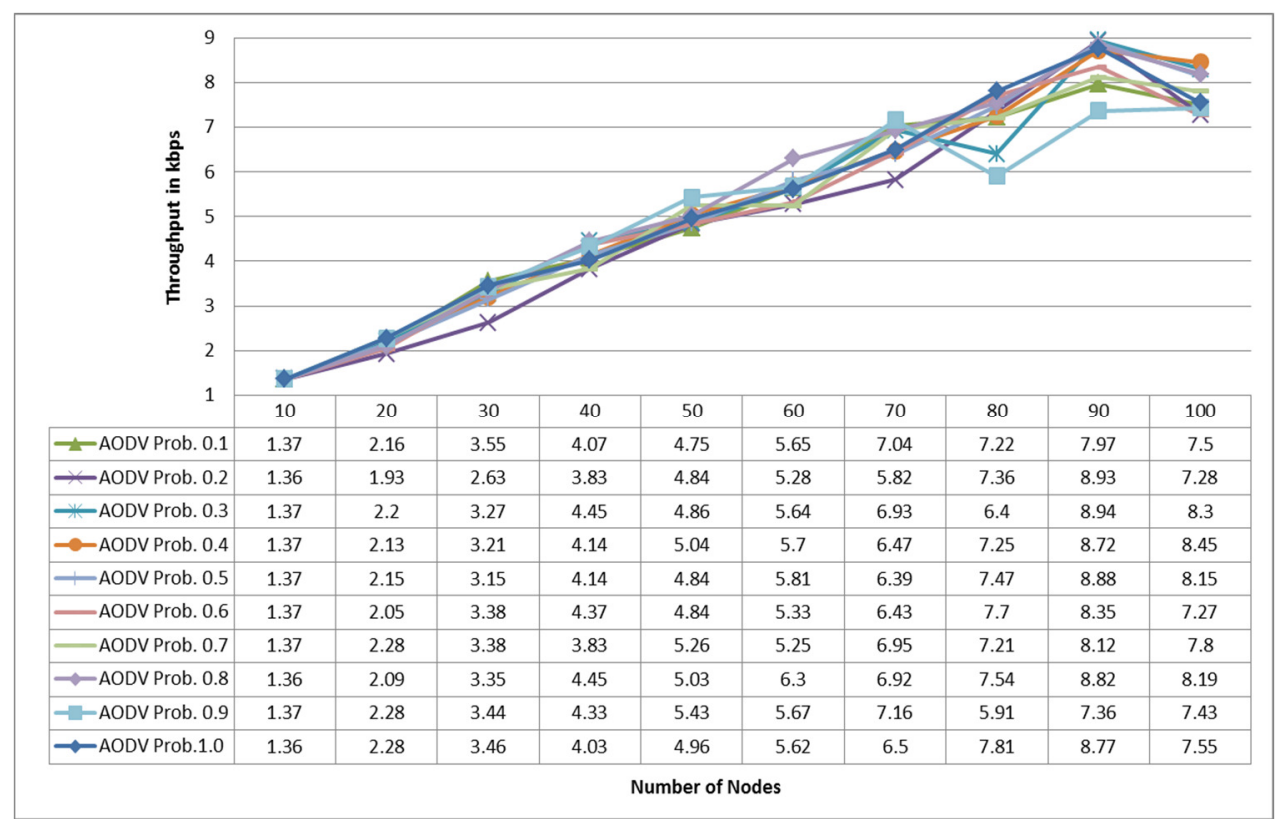

Figure 4: Average network throughput against the number of nodes for different RREQ Forwarding Probability.

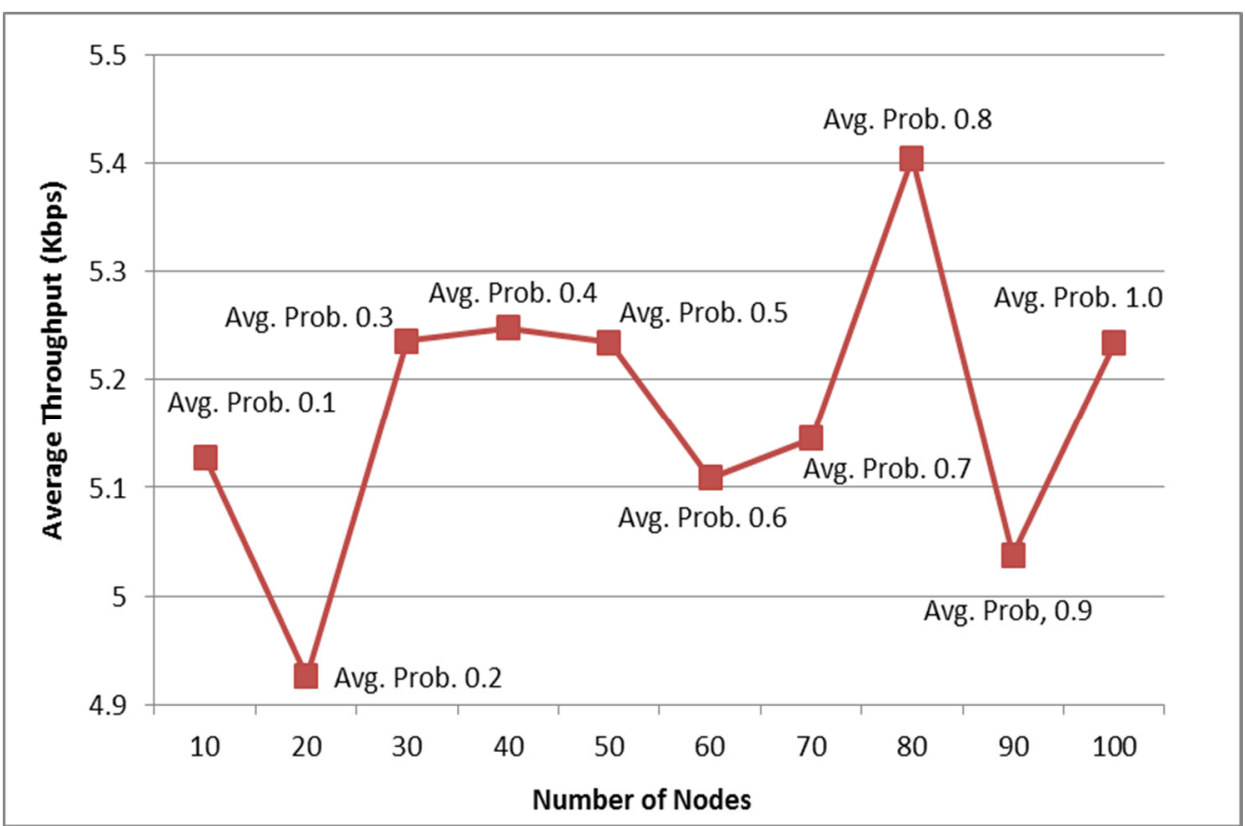


Figure 5: The average throughput against the number of nodes for average RREQ Forwarding Probability.

Figure 6 shows that the MAC Load presents very good results (is low) in small and medium size networks when the RREQ forwarding probability is 0.3 and 0.5 respectively, while for probabilities of 0.7 and 0.9 the MAC Load is very low in large scale networks. For small networks and all network sizes (Fig.(7)), RREQ forwarding probability of 0.3 provides, on average, the best performance with a MAC Load of 63.63.

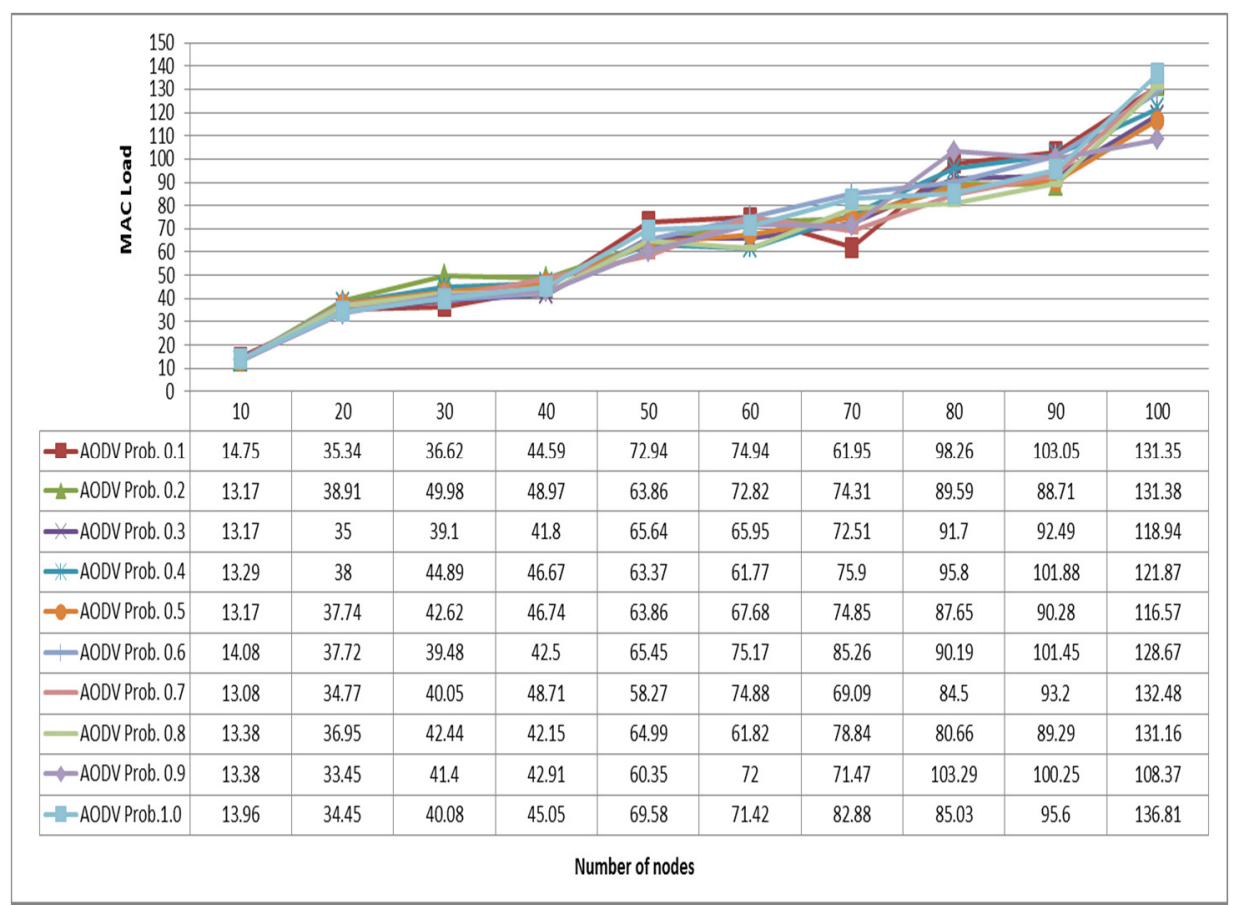

Figure 6: Average MAC load against the number of nodes for different RREQ Forwarding Probability.

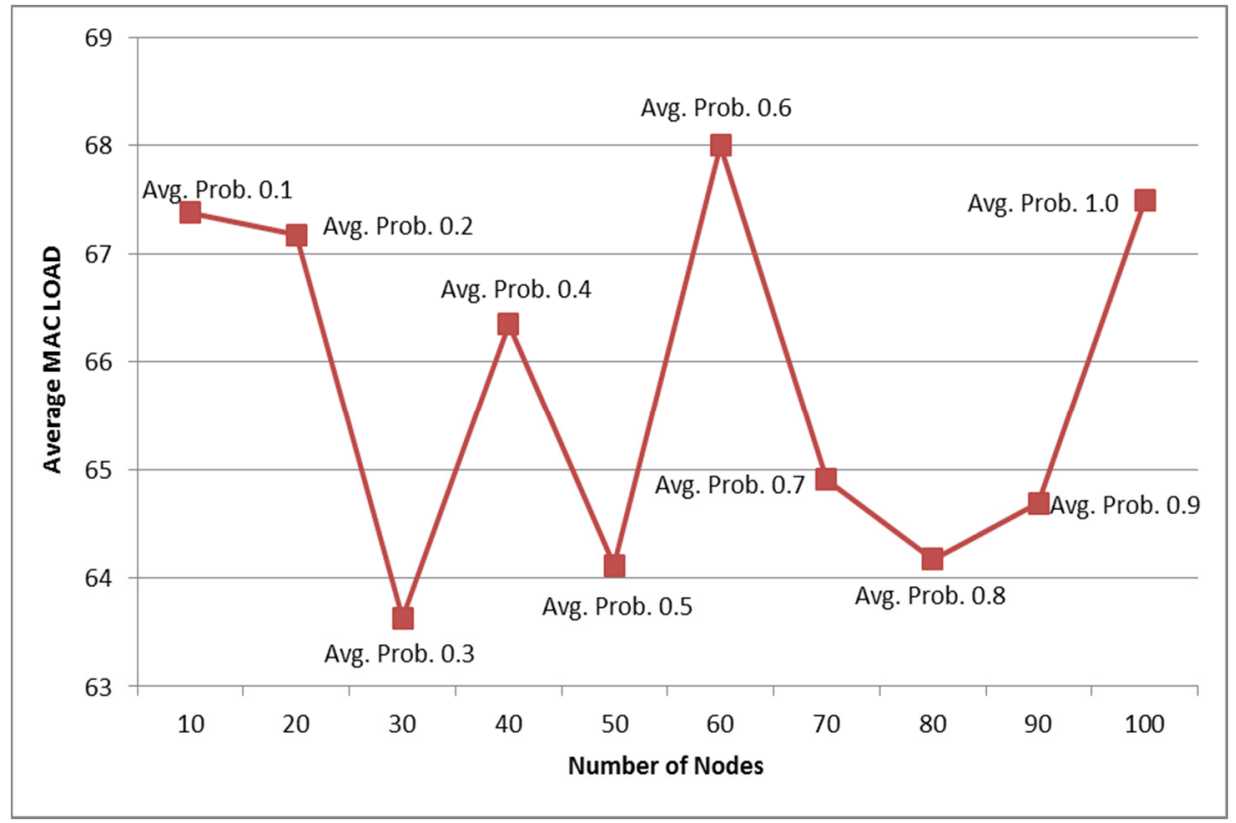


Figure 7: The average MAC Load against the number of nodes for average RREQ Forwarding Probability.

Figure 8 shows that using 0.3 and 0.5 RREQ forwarding probability in small and medium scale networks the number of dropped packets is small. Meanwhile in large scale network, the number is small for probabilities of 0.7 and 0.9 . For small networks and all network sizes (Fig.(9)), RREQ forwarding probability of 0.3 provides, on average, the best performance with the number of dropped packets as low as 70847.1.

\begin{tabular}{|c|c|c|c|c|c|c|c|c|c|c|c|}
\hline \multicolumn{12}{|c|}{350000} \\
\hline \multirow{6}{*}{ 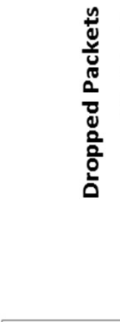 } & 300000 & & & & & & & & & & \\
\hline & & & & & & & & & & & \\
\hline & & & & & & & & & & & \\
\hline & 100000 & & & & & & & & & & \\
\hline & 50000 & \multicolumn{10}{|c|}{$\longrightarrow$} \\
\hline & 0 & 10 & 20 & 30 & 40 & 50 & 60 & 70 & 80 & 90 & 100 \\
\hline \multicolumn{2}{|c|}{- -AODV Prob. 0.1} & 23 & 245 & 2117 & 7491 & 15947 & 46298 & 62532 & 151598 & 160178 & 319311 \\
\hline \multicolumn{2}{|c|}{- AODV Prob. 0.2} & 29 & 319 & 1797 & 6940 & 14178 & 43976 & 73572 & 138237 & 153121 & 314295 \\
\hline \multicolumn{2}{|c|}{$\leftarrow$ AODV Prob. 0.3} & 33 & 317 & 2203 & 7496 & 14701 & 37312 & 68461 & 131310 & 154596 & 292042 \\
\hline \multicolumn{2}{|c|}{ *AODV Prob. 0.4} & 19 & 289 & 2560 & 7290 & 16076 & 43305 & 73330 & 151987 & 171211 & 287533 \\
\hline \multicolumn{2}{|c|}{-AODV Prob. 0.5} & 31 & 342 & 2086 & 7451 & 14178 & 46071 & 66825 & 142915 & 156193 & 297303 \\
\hline \multicolumn{2}{|c|}{-AODV Prob. 0.6} & 20 & 312 & 2107 & 7403 & 14093 & 43828 & 76706 & 156525 & 167574 & 283341 \\
\hline \multicolumn{2}{|c|}{-AODV Prob. 0.7} & 13 & 241 & 2237 & 6667 & 14597 & 44770 & 67495 & 141135 & 141559 & 293207 \\
\hline \multicolumn{2}{|c|}{ AODV Prob. 0.8} & 21 & 244 & 2304 & 7631 & 14802 & 42719 & 72428 & 126243 & 156977 & 308113 \\
\hline \multicolumn{2}{|c|}{$\multimap$ AODV Prob. 0.9} & 4 & 129 & 2272 & 6689 & 15067 & 40419 & 73754 & 145230 & 158530 & 279274 \\
\hline \multicolumn{2}{|c|}{- -AODV Prob.1.0 } & 17 & 200 & 2378 & 7500 & 16801 & 44784 & 77325 & 144006 & 158711 & 321657 \\
\hline
\end{tabular}

Figure 8: Average dropped packets against the number of nodes for different RREQ Forwarding Probability.

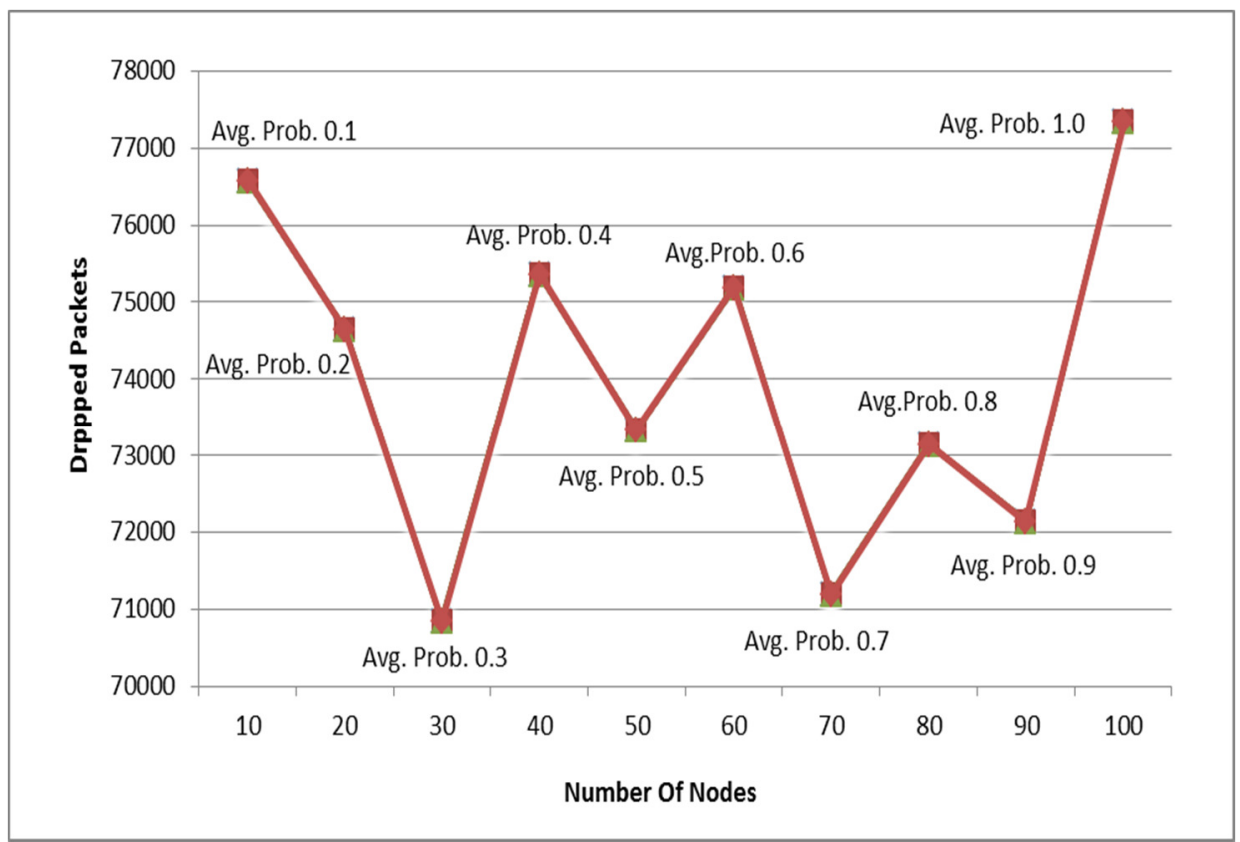


Figure 9: The average dropped packets against the number of nodes for average RREQ Forwarding Probability.

Figure 10 confirms that when the RREQ forwarding probability is $0.3,0.5$ and 0.8 the network traffic is reduced, while when the probability is $0.1,0.2,0.6$ and 1.0 the network traffic is increased. The best performance, on average, is obtained when the RREQ forwarding probability is 0.3 (Fig.(11)) which has an average network load of 41.024. Also when the RREQ forwarding probability is 0.3 the network is more reliable as it find the routes faster and is also available providing data to the sink.

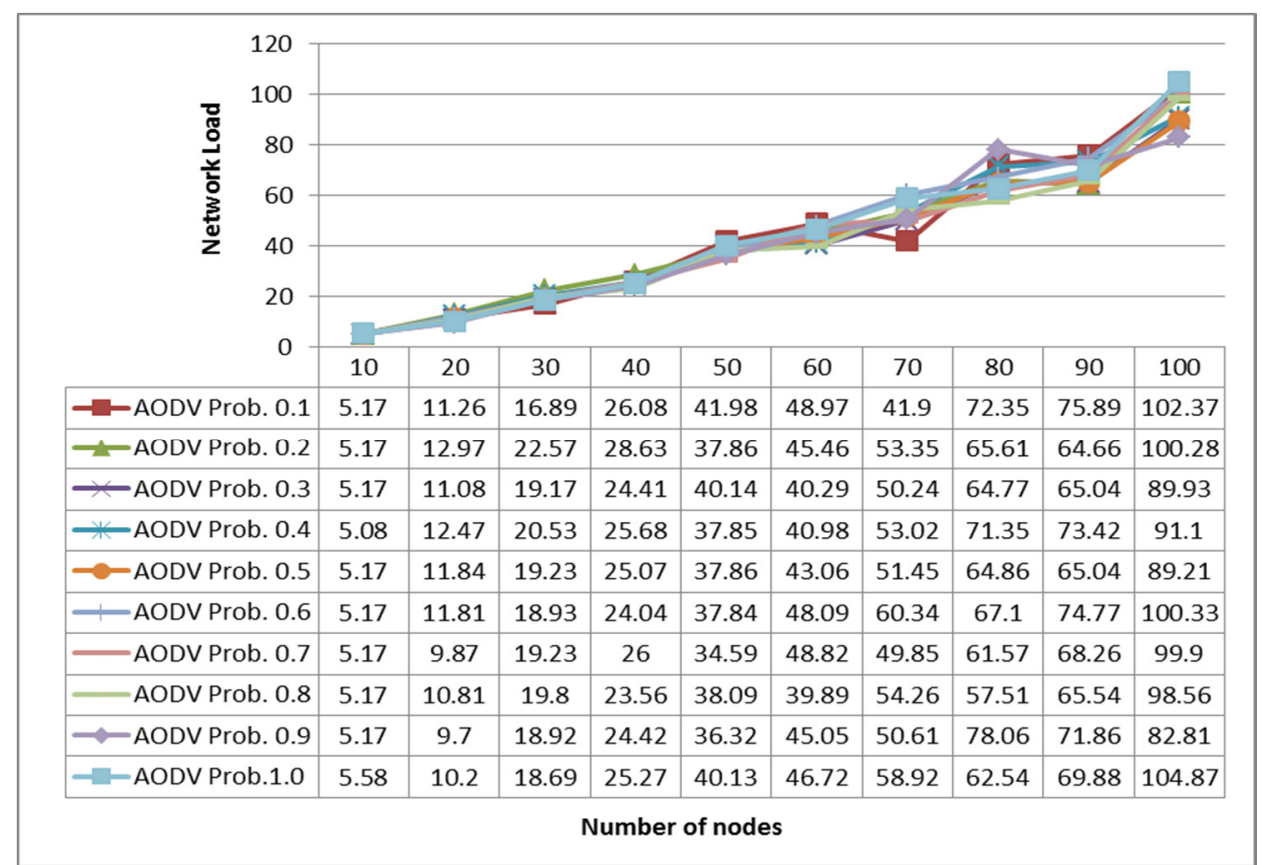

Figure 10: Average network load against the number of nodes for different RREQ Forwarding Probability.

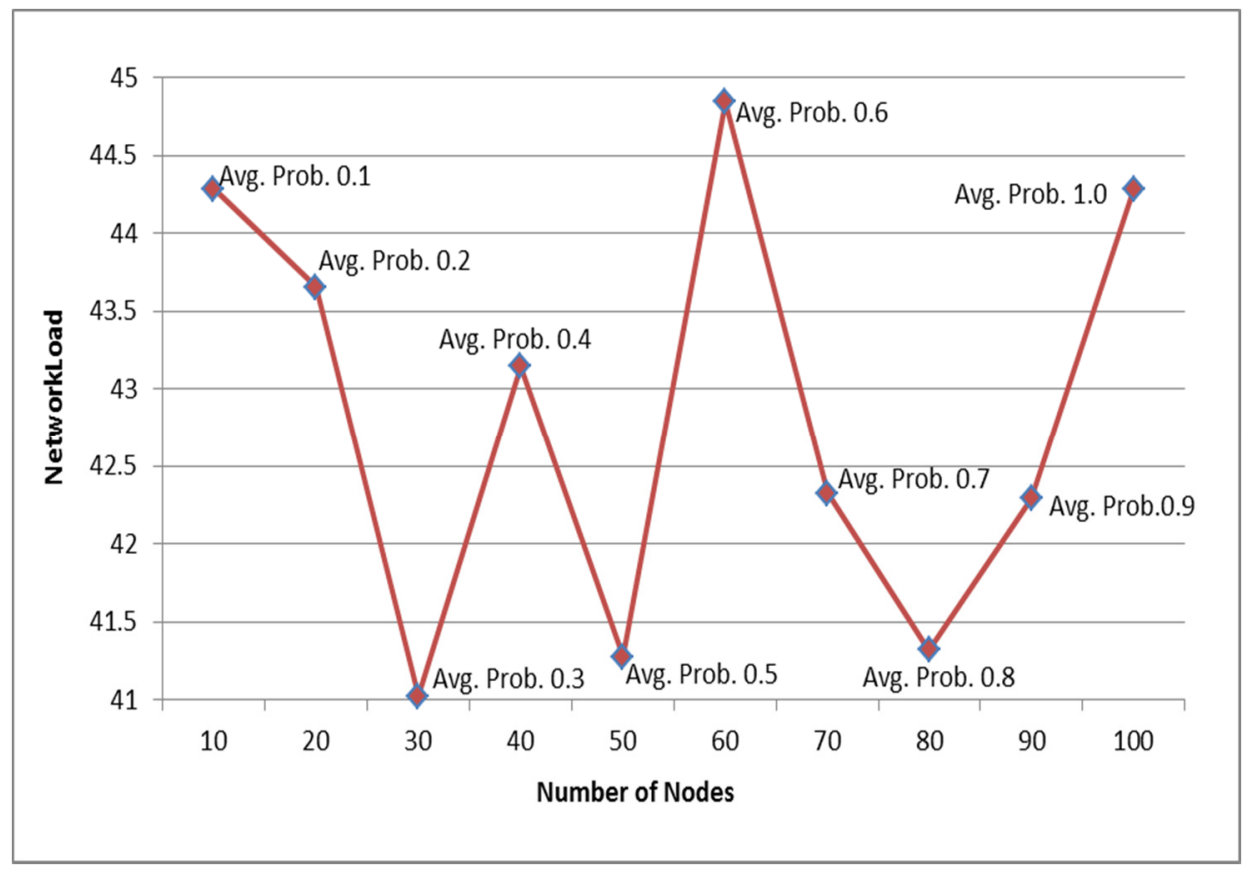


Figure 11: The average network load against the number of nodes for average RREQ Forwarding Probability.

\section{Conclusion and Future Work}

This study has evaluated AODV ad-hoc routing protocol in different message forwarding probability taking into consideration power consumption. Overall, the findings show that the energy consumption and throughput in small size networks with 30 nodes and using 0.3 or 0.4 probability did reveal very good results. Moreover for medium and large ad-hoc networks (more than 30 nodes) the probability close to 0.8 reduces the energy consumption satisfactorily. Whilst experimenting with modified AODV protocol in NS-2, using density based probabilistic route message forwarding with dynamic probability so that only a certain percentage of the n neighbours will forward the message based on the density of its neighbours will provide better results in terms of power consumption and remaining power.

\section{References}

[1].Sivanesan P., Thangavel S., (2015) 'HMM based resource allocation and fuzzy based rate adaptation technique for MANET', Optik - International Journal for Light and Electron Optics, Vol. 126, No. 3, pp. 331-336.

[2].Chonggun K., Elmurod T. and Byoungchul A. (2006) 'A Reverse AODV Routing Protocol in Ad Hoc Mobile Networks', Emerging Directions in Embedded and Ubiquitous Computing Lecture Notes in Computer Science, Vol. 4097, pp. 522-531.

[3].Verma V.K., Singh S., Pathak, N.P. (2014) 'Analysis of scalability for AODV routing protocol in wireless sensor networks', Optik - International Journal for Light and Electron Optics, Vol. 125, No. 2, pp. 748-750.

[4].Nadia Q., Fatin S. and Hamid A. (2009) 'Mobile Ad Hoc Networking Protocols' Evaluation through Simulation for Quality of Service', International Journal of Computer Science (IAENG), Feb, Vol. 36, No. 1, pp. 76-84.

[5].Giannoulis S., Antonopoulos C., Topalis E. and Koubias S. (2007) 'ZRP versus DSR and TORA: A comprehensive survey on ZRP performance', IEEE Transactions on Industrial Informatics, Feb, Vol. 3, No. 1, pp. 63-72.

[6].Johnson D., Hu Y, Maltz D. (2007) 'The Dynamic Source Routing Protocol for Mobile Ad Hoc Networks', The IETF Trust, RFC 4728, available at https://tools.ietf.org/html/rfc4728 (Accessed 01 February 2015). 
[7].Perkins, C. Royer, E. and Das, S. (2003) 'Ad Hoc On-demand Distance Vector (AODV) Routing', The Internet Society, RFC 3561, available at https://tools.ietf.org/html/rfc3561 (Accessed 01 February 2015).

[8].AbdRahman A. H. and Zukarnain Z. A. (2009) 'Performance Comparison of AODV, DSDV and I-DSDV Routing Protocols in Mobile Ad Hoc Networks', European Journal of Scientific Research, June, Vol.31, No.4, pp. 556-576.

[9].Rango F. D., Fotino M., and Marano S. (2008) 'EE-OLSR: Energy Efficient OLSR routing protocol for Mobile ad-hoc Networks', IEEE Military Communications Conference (MILCOM), pp. 1-7.

[10]. Karthikeyan N., Palanisamy V. and Duraiswamy K. (2009) 'Performance Comparison of Broadcasting methods in Mobile Ad-hoc Network' International Journal of Future Generation Communication and Networking, Jun., Vol. 2, No. 2, pp. 47-58.

[11]. NS-2 (2008) Network Simulator 2, available at http://www.isi.edu/nsnam/ns/ (Accessed 01 February 2015)

[12]. 'Bayesian Factor'(2010), May, available at http://en.wikipedia.org/wiki/Bayes_factor (Accessed 01 February 2015)

[13]. Rahman A., Islam S. and Talevski A. (2009) 'Performance Measurement of various Routing Protocol in Ad-Hoc Network', Proceeding of the International Multi-Conference of Engineers and Computer Scientist, March, Vol. 1, pp. 321-323. 